\title{
Evolution of the Cosmos and Concept of Time
}

\author{
Shouvik Sarkar \\ Independent Researcher
}

To Cite this Article

Shouvik Sarkar, "Evolution of the Cosmos and Concept of Time", International Journal for Modern Trends in Science and Technology, 6(8): 147-152, 2020.

Article Info

Received on 02-July-2020, Revised on 25-July-2020, Accepted on 02-August-2020, Published on 10-August-2020.

\section{ABSTRACT}

This article presents a new theory for the evolution of the cosmos and time. This is based on the concept of String Theory and had tried to explain the whole phenomena from the quantum level. This has also references from the mass energy equivalence theory and ether theory and so is able to explain the whole phenomena from the very beginning. It is thought that this theory can explain the evolution of cosmos and time from a completely new but more acceptable perspective.

KEYWORDS: Evolution Of Cosmos, Time Travel, Gravity, Laws Of Cosmos..

\section{INTRODUCTION}

There are many theories that claims to explain the evolution of the cosmos and none of them is completely justifiable but this theory is meant to explain the evolution of cosmos and time from almost its beginning and can be acceptable as well as it tries to explain the phenomena at the quantum level which must be the state of the universe at its early stages. So let's have a look and try to understand this theory which is named as THE THEORY OF GRAND DESIGN. First of all we should consider that the cosmos is completely designed by the nature in the form as we see today and this creation is very complex but explainable as well. We shall try to have a look at it now.

To understand the first theory that we should try to focus on is the string theory or M theory which presents the universe in the form of eleven dimensions. Next we should keep an account on the mass energy equivalence theory presented by the equation $\mathrm{e}=\mathrm{mc}$ squared and finally we should also keep focus on the ether theory which presents the movement of waves as a function of difference in potential or a ether field. These three theories is easy, have been explained and will make us understand the evolution of cosmos and also about many laws of nature like gravitation, strong nuclear force and so on. This also will make us to have a view on the dimension of time and its features.

To start with let's first understand what the dimensions are. There are literally ten dimensions in the cosmos as said by the string theory which postulates the cosmos as the multiverses which means multiple universes and each universe having six dimensions each. Let's understand the dimensions and what exactly a dimension is. A dimension is something in which movement is possible and is created only because of a potential energy difference or a field. The basic concept is that higher energy source can travel to other areas having lower energy. This is termed as potential energy difference or a field and is the reason for creation of a dimension or movement in it. Now we shall look at the ten dimensions of the cosmos.

According to the String theory there is a zero dimension which is resembled by an ideal point, first dimension is represented by a perfectly one dimensional line and can be represented by a wave 
which is an ideal one dimensional object, two dimensions are planar in nature, three dimensions are made by combination of two planes which is how we see the universe. This is because we ourselves are three dimensional beings so our perception makes us to view the world as a three dimensional object, fourth dimension is time, fifth dimension is travelling forward and backward in time, sixth dimension is having two existences of a single object and the dimension by which they can meet. Generally there are six dimensions in an universe but the cosmos is made of multiple universes or multiverses. So the seventh, eighth and ninth dimension is said to be the dimensions that connects the universes in the cosmos. Tenth dimension is the time in that place of the cosmos. This is how the ten dimensions of the cosmos is lined up. Each has different characteristic fields due to which movement in either of them is in different ways. But at first there was enormous amount of energy and negative energy was existent in the cosmos. From where that came is a mystery so the concept of eleventh dimension came according to $\mathrm{M}$ Theory as per which the energy and negative energy originated from eleventh dimension through dimensional travel. In eleventh dimension it may have been in any other object form which could not be found out.

Now in the cosmos this energy and negative energy originated in vacuum. If we combine these two the vacuum is created back. Fortunately this event had occurred only once so far in history due to which total energy of the cosmos is conserved and is zero which also gives rise to the concept of fixed events of time as every phenomenon afterwards is only automated. Now as the energy and negative energy arrived it started travelling in vacuum space as the surrounding is having no or zero energy hence there always had been an energy gradient or field which is said as the ether field. Now negative energy is something exactly as energy but opposite in amplitude to energy. This is why when we combine them zero energy or vacuum is created back.

Now that energy started travelling in vacuum in the tenth, ninth, eighth and seventh dimensions of the cosmos. In cosmological time dimension it travelled only in one direction and so from the point onwards on which the energy originated afterwards is regarded as positive time. Keep in mind that the measure of cosmological time, time in some other universe and that in our universe can be different. Hence the energy travelled as waves in time dimension due to this energy gradient or ether field. The waves are nothing but ripples of energies in an alternate fashion as it travels through the medium. The energy also travelled in the three dimensional cosmological space due to the field. But the initial source of energy was sound energy which had higher wavelengths and these sound waves are still found in the cosmos. These sounds are often heard in the cosmological space. Sound is nothing but how the organism audio organ behaves to the high frequency sound waves and later captured and stored by the brain to process it. This topic will be discussed in my subsequent articles.

But sound waves are not what gave rise to particles. It's the light waves. The conversion took place in those early stages when various different type of waves originated from out of phase interference of sound waves with itself, followed by with newly formed waves which are formed by partial interference only, that are micro waves, infra red waves, ultra violet ray, gamma ray and $\mathrm{x}$ rays. In some of these interference patterns give rise to visible light waves while other gave rise to other different types of waves. In this way light waves were generated. We should also note that in phase interference will either increase the amplitude or lower it or even may make it vacuum but could not change the waveform. The interference took place because the energy originated from different position in the cosmos and had travelled all across in spherical directions. This makes us assume that the cosmos is spherical in shape and infinite in dimensional length but no proofs have been found of it yet.

Now the cosmos at that stage had all sorts of waves including light waves. We have also found that if we collides two photos then an electron, a positron and quarks are emitted. These are the most elementary particles of nature. In fact while electron is a particle positron is considered as anti particle. Our basic elementary structures are not comprised of positron rather electrons and protons $\&$ neutrons (which are composed of quarks) which are called particles and the subsequent element is called matter. There can be universes or structures in the cosmos made of anti particle and hence anti matter. There are some other things called dark energy and dark matter. They are something we can't see. Dark energy is all other energy except light while dark matter is matter that is made of waves other than light. In fact particles derived from light waves reflect light and hence our sense organ detects it. But matter derived from waves other than light does not reflect light and hence cannot be seen. So it's called dark matter. These 
are the concepts of various types of objects found in the cosmos. Now we shall move to the evolution.

There can be bodies made of these anti matter or dark matter as well but our universes hardly have something like that. Now we shall look at how the electrons, protons and neutrons are formed. Electrons are nothing but energy revolving in very small orbits. It is visible as it is made of light waves and it has mass as most of the energy is concentrated at a small region and hence considered to be stationary. In fact mass is nothing but very high amount of stationary energy. The mass of an object is given by the energy mass equation of Albert Einstein. So if $\mathrm{E}$ energy is concentrated at a point then its mass is given by $\mathrm{E}$ / c squared. This is how the composition of most elementary particles that is electrons, positrons and quarks are. Now the charge of these particles depends on the amplitude and direction of the energy. For a positive amplitude part of energy the charge will be positive while for negative amplitude energy it would be negative. The spin of these particles depends on the direction these energies revolve. This is how the mass, charge and spin of these elementary particles emerged. Now let's see how the energy started revolving in orbits. When two light waves collided at angles then that made the energy to start revolving. This is due to the fact that light waves reflect each other as similar to two balls colliding and hence the energies started revolving in orbits.

Now we shall see that the elementary particles thus formed has a lifetime which is though very large even more than lifetime of the universe. This is because these revolving waves are always losing its energy because some of this energy travels in the perpendicular direction to the radius at which the energy is at that moment. This energy is though very small but is also the reason for electromagnetic force. Now we shall see the reason for electromagnetism, gravitation and strong nuclear force. When a wave is rotating it is at a point in the orbit at a time, The other portions of the orbit is having nothing and hence any energy would like to travel to that position be it from this particle or other. This is the reason of gravitations. In fact other particles are attracted to that portion and some revolving energies are emitted towards those places before the particle reaches to that place exactly similarly as an energy is radiated out comprising the electromagnetic force. These revolving energies (particles) are called gravitons. Now if the amplitude of the revolving waves of two particles are same say positive or negative there will be no energy gradient hence they do repel each other as it would try to move towards energy gradient places rather places with similar energy. But if a positive energy is taken towards a negative energy it will attract each other because there will be a energy gradient. This is how the electrostatic forces work. So we have discussed the reasons for electrostatic, electromagnetic and gravitational forces. Now we shall have a look at the strong nuclear force.

The particles in the cosmos were been already moving and hence they had a momentum. This is because in spite of moving in orbits the energies had also a linear momentum so the so formed particles in spite of rotating also have been moving in straight lines in space. Now due to this force and gravitation two or more quarks were been attracted together overcoming the electrostatic repulsion. Though the repulsive force is more than the gravitational forces still the previously gained momentum makes it to overcome and get bind with another particle with their energy orbit crossing each other twice. But they never collide because their speed is same and they are not at the same position at the same time. Thus they are bound in a form of equilibrium. This is because at so close place the gravitational pull becomes more than the electrostatic repulsion. Hence the two particles get bound by strong nuclear force. When two up quarks and a down quark is bounded a proton is formed while when two down quark and an up quark is bounded it forms a neutron. Then the protons and neutrons also get bounded in a similar fashion and hence the nucleus of the atom is made. Different other types of combinations are also possible but they are not stable and hence they destabilize to its basic particles back.

Now in atoms the electrons revolve in orbits around the nucleus. This happens as when these electrons are coming closer to the nucleus in different lines of travel it started falling over it due to electrostatic and gravitational attraction but never in fact falls rather start revolving due to the initial momentum it had. In fact among many possibilities there is only one combination that makes an atom. These electrons in the atoms also falls slowly towards the nucleus but its lifetime is too very high. Then two atoms combine to forms molecules and so on slowly giving rise to stars in the cosmological space. This is how the primary celestial bodies in the cosmos are formed. Next we shall look at the creation of the universes and dimensions in it. Remind there can be many different types of particles or matter too that have 
existed in the cosmological bodies but they have also been created similarly and can be understood.

Now we shall look after how the dimensions got created. So we had a cosmos in which there were celestial bodies, huge amount of energies and free particles roaming in the cosmos. Now before understanding the creation of dimensions we should try to understand time in a better manner. For that even before we should understand the travelling of dimensions. As observations suggests and also supported by string theory dimensional travel is possible through wormholes. Wormholes are present everywhere in the cosmos but through these wormholes only one dimensional object that is waves can pass through. So for a object to travel through wormhole it is required that the object is converted back to energy which can be accomplished by variety of means like providing that much energy to the object so that its bonds are disintegrated and it is converted back to energy or by moving it with speed of light as suggested by Albert Einstein. Once converted to energy or one dimensional object it can travel higher dimensions. The method of converting objects or particles into one dimensional object that is energy and its transportation into other dimensions is called teleportation.

In fact the light waves or sound waves or even electromagnetic waves always travel from past or future and hence can be detected by human brain as per brain functionality which is an universal wave detector. This process is first called Déjà vu by the Chinese and hence is what is given its name. This gives us a brief idea that future events, past events and present events exist simultaneously and make us think time in a new way.

The concept of time is based on like this. Whenever an object travels through time some of its energy stays stored in the past while the rest travels with time. This is why the energy of each and every object decreases with time. Though this energy decrease is very low and so it can't be detected easily. The rest of the energy traverses while it may gain or loose energy to and from its surrounding as well as it travels. The speed at which these objects travel through time is much more as compared to our consciousness which too travels with time. This means when we experience the present, events may have taken place much in the future and also the past, present and future exists simultaneously hence the possibility of time travel also stands. Now we have seen that in the time dimension the objects exists throughout it in original form (as that part of energy stays in past while the object passes through time) and hence if we travel through time dimension along with our consciousness at a faster speed in forward or backward direction through teleportation then we can travel forward or backward in time and hence time travel would be possible. This is the concept behind time travel. This is how the cosmos have worked itself and also we should keep in mind that every event in the cosmos is automated and hence each and every event is fixed from before. This is supported in cosmological dimensions as well in the universal dimensions on various universes in the multiverses cosmos.

Now we shall look first at how the fundamental forces work for bigger objects. Let's start with gravitation. The force works similarly for larger objects as for smaller objects. But the total force exerted by larger objects is much more as compared to that of smaller objects due to accumulation of huge number of small particles. The net force sums up. Now according to gravitational theory both the objects will exert a force on one another which can be easily shown by this model explaining quantum gravitation. Now the smaller particles will be attracted by a more force due to the energy gradient, mass of the larger particle and it have a chance to get bounded to the larger objects. But a perfect binding does not occur due to the repulsion of the particles at surface of both the objects which is due to the reason of presence of higher molecular structures which are more compact and hence prevents binding of particles. But the smaller particles of the bigger objects and also that of smaller objects still attracts each other due to which a gravitational force is always exerted on both the objects and hence they stay together. This is the basic concept behind gravitation. Let's explain it a bit more deeply.

In solid particles the atoms are very tightly packed and hence there is no affinity for bounding. Though at quantum level there is yet an energy gradient which is the reason for gravitation but before reaching there would always be repulsive force acting due to electromagnetism (net force is zero) and hence two electrons which are in the outer orbits of an atom can never combine. But at particle level there will always be a possibility that gravitational force wins over the electrostatic force and hence will disintegrate the atom. This is why molecules are formed which and hence two solid particles stays over one another because they have no affinity for each other. But still the gravitational forces are exerted at gravitational level and more the no of particles more is the force exerted due to 
higher affinity. This is why lighter particles are attracted towards heavier particles as explained earlier. With electrostatic attraction or repulsion the case is also same. It do acts similarly though it may somewhat cancel out if opposite charges are available in the same object or add up if similar charges are available in the object. Hence a net electrostatic force is sometimes present from the objects only. But for the strong nuclear force the force acts only if both the particles can come closer which is only possible if it has an initial very high momentum. But for general objects that is not seen hence for larger objects the two particles of an atom never come too much closer and hence strong nuclear force is obsolete, as it will create an impact only if both can make a stable binding at the quantum level. This is how these forces shape up for higher objects. Now in the cosmos in this way higher molecule, even higher objects like stars celestial bodies, galactic oceans and many other objects are formed with having these basic forces of the cosmos acting.

Now we shall have a look at the creation of the dimensions of the universe and later its creation. As we know dimension is what difference in potential or field. So if we change the difference in potential to zero then the movement in that dimension can become impossible. This is same with time as well as the three dimensional space. Like if there is object with more mass than us in space then we cannot move pass it. This actually means that object has more energy than us but if that is it then it should pass over us. But in fact the other forces acting on it makes its energy equal to that of its surrounding and hence it stays stagnant. But our energy is too equal to that of the surrounding when we approach it. If we exert more force on the object then the surrounding also exerts more force on that object due to which the energy of both stays same which is equal to the surrounding. This is why it does neither move nor can we go through it. But if we can provide it enough energy and the energy we have can be more than that of the object then a energy gradient will be created and we can travel through it by moving it. But in that case we travel slower through time. In fact more energy we use the speed at which we travel time is lesser. In fact we are always loosing some energy due to travelling through time. This is another basic law of the cosmos. Now let's see that how higher dimensions in the cosmos are created.

Say in our universe there are six dimensions, in fact four general dimensions and two dependent dimensions as described above. How these dimensions got created is yet what I could not find out but the concept of the multiverses is what I can explain. It's like for our universe the dimensions are again infinite for space and time hence if we travel in three dimensional space we can never get out of it. But to travel to other universes or the cosmological space we have to convert ourselves into waves by teleportation $\mathrm{s}$ demonstrated by String theory. Now the laws in our universe is same as that in the cosmos except the speed at which we travel through space and time in it is different from other universes and cosmos. There also in a similar fashion stars, planets, asteroids, comets etc are formed. In fact comets are nothing but planet like objects which couldn't find an orbit and so travels freely in the universe due to energy gradient. But planets started revolving around stars or satellites around planets (in fact a smaller body around a larger body) as follows, The planets was at a stage moving in the universe with a speed but due to strong gravitational pull of the stars it gets attracted and started falling over it eventually forming orbits around it. In this way the solar systems were formed. All the things hence formed is formed due to one required combination among millions of possible combinations but these required combinations formed are huge due to presence of infinite or very large possible combinations. Hence we see what we see around us such as galaxies, solar systems, stars, planets etc. Mechanism of time travel is also similar in our universe as that in the cosmos. In this way the laws of our universe are formed.

Now we shall have a brief look here at the development and mechanism of humans and how everything in the cosmos is fixed as its fate or destruction. Humans are nothing except a creation by the cosmos which functions as defined by the cosmos. We know human works according to its thinking which is provided by the brain signals or waves that are generated in the brain and hence defines the whole working of humans. But these brain waves are a result of its previous works and thinking. The thinking of human brain is affected from the electromagnetic waves from the planets, stars or any other object but from planets and stars is what is significant due to their size. Hence there thinking is predefined as its working and so forth its future works. This is similar for all organisms and hence all interactions, everything is as a result of the location of the stars and is a field of study of astrology. This will be described more elaborately in my subsequent articles. But the positions of the stars, planets are also fixed as considered from the 
situation of creation of the cosmos and hence predefined. Hence activities and life of humans are also predefined. Thus everything in the cosmos is predefined and is a result of the point of creation of energy as afterwards all phenomena is automated. So everything the beginning of the universe, fate of objects like stars, planets etc or humans or even the end of the cosmos can be calculated. This is another very big finding of science and a major field of study.

The human body can be thought as a machine also which is governed by the laws of physics like we can move a hand because of blood and bone in it. The blood has iron which on getting electrical impulses through neurons moves exactly as a motor does and hence we can move our hand. Again for viewing our eye acts as a camera in which the light waves are captured. In fact when a light wave falls on an object it absorbs the light wave and then go to an excited state. To come back to normal state it emits a photon and hence that photon is detected by our eyes. The eye acts as a camera and the brain process it so that we are able to see it. This is how our various body parts works. Hence we are amazing creation by nature which has our keys.

Now the end and beginning of the objects in the cosmos will keep on going as the particles looses energy and hence it will definitely collapse one day. But new particles will again be formed and new universes will keep on forming. This is how the cosmos will keep on going on. But much before that our universe and solar system will get destroyed due to various phenomena we already know and thus this ever ongoing cycle of creation and destruction will keep on going. But what we conclude from this article is the cosmos is the result of a grand design by the nature.

\section{ACKNOWLEDGEMENTS}

I would like to thank all the people whose works became the baseline of my work like Sir Albert Einstein, Nikola Tesla and Dr Michio Kaku. I would also like to thank all who supported me in it.

\section{REFERENCES}

[1] Albert Einstein. Mass Energy Equation, Wikipedia.

[2] Michio Kaku. String Theory, Wikipedia.

[3] Nikola Tesla, Ether Theory, Harvard.Edu 